FRI0671

ADAPTATION AND VALIDATION OF THE ANKYLOSING SPONDYLITIS QUALITY OF LIFE (ASQOL) QUESTIONNAIRE FOR USE IN SERBIA

M. I. Zlatkovic-Svenda ${ }^{1}$, M. M. Radak-Perovic ${ }^{1}$, J. Milicevic ${ }^{2}$, R. Stojanovic ${ }^{1}$, B. Lazovic ${ }^{3}$, P. Ostojic ${ }^{1}$, J. Wilburn ${ }^{4}$, S. McKenna ${ }^{5} .{ }^{1}$ Institute of Rheumatology, University of Belgrade School of medicine, Belgrade, ${ }^{2}$ Hospital Center Uzice, General hospital Pozega, Uzice, ${ }^{3}$ University Clinical Hospital Center Zemun, University of Belgrade School of Medicine, Belgrade, Serbia, ${ }^{4} 4$ Galen Research Ltd, ${ }^{5}$ School of Health Sciences, University of Manchester, Oxford Road, Manchester, United Kingdom

Objectives: To translate and adapt a Serbian version of the Ankylosing Spondylitis Quality of Life (ASQLL) questionnaire and to validate it in Serbian speaking AS patients.

Methods: The ASQoL development was conducted in three stages. The first stage comprised of a dual-panel translation: a bilingual panel (to provide the initial translation into the target language) and a lay panel (where items are assessed by people of average and below average education levels for comprehension and "naturalness" of language). The second stage involved cognitive debriefing interviews to assess the applicability, relevance and comprehensiveness of the new questionnaire. The third stage was a psychometric evaluation of the new questionnaire to assess construct validity, convergent validity and known-group validity. Convergent validity determined associations between ASQoL and NHP section scores by using Spearman's rank correlation coefficients. Known-group validity was evaluated by testing of the ASQoL ability to distinguish between groups of patients who differed according to perceived general health, incidence of flare-ups and perceived disease severity. Nonparametric tests for independent samples (Mann-Whitney U test for two groups or Kruskal-Wallis one-way analysis of variance for three or more groups) were used. Internal consistency was assessed using Cronbach's alpha coefficient. Reproducibility was measured by test-retest reliability.

Results: Cognitive debriefing interviews were conducted with 10 AS individuals (50\% male), aged $38,4(8,8)$ years. A few items (i.e. chronically and 'I get frustrated...') were not fully understood by all participants and were amended to be more natural sounding in Serbian. The Serbian ASQoL was deemed relevant, clear and easy to complete. Psychometric evaluation was conducted in 60 patients $(70 \%$ male), aged $37,9(11,1)$ years. No significant differences in ASQoL scores were found between males and females or between older and younger patients $(p=0.41, p=0.16)$. Considering convergent validity, the ASQoL correlated the most strongly with the Pain $(r=0.79)$ and Emotional reactions $(r=0.78)$ sections of the NHP. The ASQoL highly correlated with the Physical mobility $(r=0.77)$ and Energy scales $(r=0.75)$, indicating the importance of these factors on QoL in AS. The ability of the Serbian ASQoL to detect meaningful differences was demonstrated by ASQoL scores according to patients perceived general health $(p<0.05)$ and disease severity $(p<0.05)$. Cronbach's alpha coefficient for the ASQoL was 0.95 at Time 1 and 0.91 at Time 2, indicating good internal consistency. Testretest reliability was good, with a correlation coefficient of 0.84

Conclusions: The Serbian version of the ASQoL demonstrated good psychometric properties proving it to be a valid and reliable tool for use in routine clinical practice and in clinical trials.

Disclosure of Interest: None declared

DOI: 10.1136/annrheumdis-2018-eular.4012

\section{FRI0672 ANTI-SACCHAROMYCES CEREVISIAE ANTIBODIES IN SPONDYLARTHROPATHIES: PREVALENCE AND ASSOCIATIONS WITH DISEASE PHENOTYPE}

R. M. Ferreira ${ }^{1}$, F. Aguiar ${ }^{1}$, T. M. Rocha ${ }^{1}$, S. Ganhão ${ }^{1}$, A. Águeda ${ }^{2}$, M. Guerra ${ }^{3}$, S. Pimenta ${ }^{1}$, M. Bernardes ${ }^{1}$, L. Costa ${ }^{1}{ }^{1}$ Rheumatology, São João Hospital Centre, Porto, ${ }^{2}$ Rheumatology, Centro Hospitalar Baixo Vouga, Aveiro, ${ }^{3}$ Rheumatology, Centro Hospitalar Vila Nova Gaia, Gaia, Portugal

Background: It has been speculated that increased gut inflamation is of aetiopathogenic importance in the development of Spondylarthropathies (Spa). Serological markers, as anti-Saccharomyces cerevisiae antibodies (ASCA), which are rarely positive in healthy controls $(<5 \%)$, possess clinical significance in inflamatory bowel disease (IBD) management. ${ }^{1}$ Because Spa and IBD share similarities and even subclinical intestinal inflamation may be present in a high number of Spa patients, evaluation of this antibodies has gained increasing relevance.

Objectives: To investigate the status and frequency of ASCA in Spa patients and the association of these serological markers with the clinical profile.
Methods: We performed a retrospective study including SpA individuals treated with biologic therapy, followed at our Rheumatology department. Classification of $\mathrm{SpA}$ was based on the ASAS criteria. Medical records were obtained by consulting the national database (Reuma.pt). ASCA IgA and IgG levels were measured in the period time between 2016 and 2017 and determined by ELISA. The quantitative ASCA results were expressed in $\mathrm{RU} / \mathrm{ml}$ and 20 was established as the cutoff point. $\mathrm{X}^{2}$ or Fisher tests were used for analysis of categorical variables and $\mathrm{t}$ test or Mann-Whitney for continuous variables (SPSS 23.0). The adopted significance was of 0.05 .

Results: We included 231 Spa patients, 117 of which were men $(51 \%)$, with a mean age of $48.6 \pm 12.5$ years. The median disease duration was 17 years [min:2; max: 53 ]. In total, $39 \%$ of the patients had isolated axial form $(n=90), 10 \%$ isolated peripheral form $(n=23)$ and $51 \%$ presented axial and peripheral involvement $(n=118)$. Nine patients had associated IBD [7 cases with Crohn Disease (CD) and 2 with Ulcerative Colitis (UC)] and 66 patients presented concurrent psoriasis (28.6\%). Ninety-three patients (40\%) were HLA-B27+ and 59 (26\%) presented history of uveitis (current or previous). ASCA IgA were positive in $14 \%$ of the whole sample ( $n=33 ; 14$ patients with isolated axial form, 4 with isolated peripheral form and 15 with axial and peripheral form). ASCA IgG positivity was found in $5 \%$ of the Spa ( $n=12 ; 7$ patients with isolated axial form, 1 with isolated peripheral form and 4 with both forms). The median ASCA IgA and IgG titers were $72 \mathrm{RU} / \mathrm{ml}$ [min:22;max:200] and 45.5 RU/ml [min:28;max:200], respectively. We found no statistically significant difference in the number of ASCA IgA or IgG-positive patients in $C D$ vs UC $(p=0.722 ; p=0.583)$. Current age and at diagnosis, disease duration, gender, active or past smoking habits were similar between ASCA IgA or IgG-positive and negative groups. Also, disease phenotype including peripheral arthritis, axial involvement, psoriasis, HLA-B27 positivity and uveitis were unrelated to ASCA IgA and $\lg G$ status.

Conclusions: Our results showed that Spa patients presented an increase of ASCA IgA positivity, in agreement to previous data. No relationship of ASCA status was found with the demographic aspects or clinical presentation. In the future, our purpose is to investigate the relationship between antibody reactivity and endoscopic findings.

\section{REFERENCE:}

[1] Peeters M, et al. Diagnostic value of anti-Saccharomyces cerevisiae and antineutrophil cytoplasmic autoantibodies in inflammatory bowel disease. Am J Gastroenterol 2001;96:730-4.

Disclosure of Interest: None declared DOI: 10.1136/annrheumdis-2018-eular.2790

\section{FRI0673 DO PATIENT REPORTED OUTCOME MEASUREMENT INFORMATION SYSTEM (PROMIS) COMPUTER ADAPTIVE TESTS CORRELATE WITH DISEASE ACTIVITY IN JUVENILE IDIOPATHIC ARTHRITIS?}

R. Trachtman ${ }^{1}$, E. T. Murray ${ }^{1}$, J. Szymonifka ${ }^{1}$, A. B. Adams ${ }^{1}$, N. Pan ${ }^{1}$, S. F. Taber ${ }^{1}$, K. B. Onel' ${ }^{1}$, L. A. Mandl' ${ }^{1}{ }^{1}$ Hospital for Special Surgery/Weill Cornell Medicine, New York, United States

Background: The importance of patient-reported outcomes is increasingly recognized both in clinical care and in research. PROMIS is an NIH-supported collection of patient-reported outcome measures, covering a variety of domains that are designed without disease specificity. While 'short forms' have been studied in juvenile idiopathic arthritis (JIA), PROMIS computer adaptive tests (CATs) have not.

Objectives: This study evaluates whether PROMIS CATs correlate with disease activity in patients with JIA.

Methods: A convenience sample of patients with JIA $(N=21)$ were recruited from a single center. Patients aged 10-17 years completed all available pediatric PROMIS CATs, and parents of patients aged 2-9 years completed all available parent proxy PROMIS CATs (fatigue, pain interference, peer relations, anxiety, depressive symptoms, and mobility). Correlation of the CATs t-scores with disease activity, as measured by the Juvenile Disease Activity Score-71 (JADAS71), (0-101, higher being worse) was evaluated using Spearman correlation coefficients.

Results: All families approached completed the PROMIS CATs: 13 patients and 8 parents (table 1). Median age was 12.7 years (range $1.3-18.6$ years), and mean JADAS-71 score was 9.58 (SD 2.07). 69\% of patients completed PROMIS CATs remotely via smartphone. Anxiety $(r=0.74, p=0.006)$, depressive symptoms $(r=0.84, p<0.001)$, and pain interference $(r=0.64, p=0.018)$ CATs correlated 
strongly with JIA disease activity (table 2). Among parent proxy CATs, only anxiety correlated with disease activity $(r=0.71)$; however the association was not statistically significant.

Table 1 Patient characteristics

\begin{tabular}{ll}
\hline Characteristic & $\mathbf{N}=\mathbf{2 6}$ patients \\
\hline Age, years, median [IQ range] & $12.7[6.0,14.5]$ \\
Male & $8(30.8 \%)$ \\
$\begin{array}{l}\text { White or Caucasian } \\
\text { Insurance }\end{array}$ & $20(76.9 \%)$ \\
Medicaid & $6(23.1 \%)$ \\
Device & \\
Smartphone & $18(69.2 \%)$ \\
iPad & $3(11.5 \%)$ \\
Location & \\
In hospital & $8(30.8 \%)$ \\
Remotely & $18(69.2 \%)$ \\
\hline
\end{tabular}

Table 2 Spearman correlation coefficients for PROMIS domains and JADAS71 score

\begin{tabular}{ll}
\hline PROMIS domain & JADAS71 score Spearman correlation coefficient \\
PATIENT SCORES & \\
\hline Fatigue T-score & 0.488 \\
Pain Interference T-score & $\mathbf{0 . 6 4 0}$ \\
Peer Relations T-score & -0.345 \\
Anxiety T-score & $\mathbf{0 . 7 3 8}$ \\
Depressive Symptoms T-score & $\mathbf{0 . 8 4 0}$ \\
Mobility T-score & -0.671 \\
\hline
\end{tabular}

Conclusions: Our results demonstrate that the PROMIS CATs are feasible to administer in an outpatient pediatric rheumatology setting. Anxiety, depressive symptoms, and pain interference were significantly correlated with disease activity, even though mean disease activity was relatively low. This underscores the negative effect on quality of life of even mild disease. Parent proxy CATs showed poor correlations with disease activity, suggesting parents are inaccurate in assessing important aspects of their child's health. Larger prospective studies are needed to evaluate the sensitivity of PROMIS CATS to change in disease activity over time.

Disclosure of Interest: None declared

DOI: 10.1136/annrheumdis-2018-eular.5743

\section{\begin{tabular}{|l|l}
\hline FRI0674 APPLICABILITY OF THE PSAID12 QUESTIONNAIRE AS \\
\hline
\end{tabular} A CORE OUTCOME MEASUREMENT IN PSA CLINICAL TRIALS: AN EVALUATION USING OMERACT FILTER 2.1} INSTRUMENT SELECTION CRITERIA

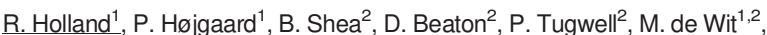
W. Tillett ${ }^{1}$, R. Christensen ${ }^{1}$, N. McHugh' 1 , P. Mease ${ }^{1}$, A.-M. Orbai ${ }^{1}$. ${ }^{1}$ Group for Research and Assessment of Psoriasis and Psoriatic Arthritis, ${ }^{2}$ Outcome Measures in Rheumatology, International Organisation, -

Background: The Group for Research and Assessment of Psoriasis and Psoriatic Arthritis (GRAPPA)-Outcome Measures in Rheumatology (OMERACT) Psoriatic Arthritis (PsA) Core Set working group is in the process of selecting core outcome measurements for PsA randomised trials, following OMERACT Filter 2.1 for instrument selection ${ }^{1}$. The Psoriatic Arthritis Impact of Disease questionnaire (PsAID12) passed the first two steps (domain match and feasibility) at the GRAPPA 2017 annual meeting and is a candidate instrument to measure PsA specific health related quality of life (HRQOL)/Life impact.

Objectives: To conduct a systematic review (SR) of the PSAID literature to review the measurement properties in the OMERACT Filter 2.1, assess specifically construct validity (truth) and discrimination of the PsAID12 questionnaire based on available evidence, and to identify gaps in knowledge that need to be covered in order for PSAID12 to pass Filter 2.1.

Methods: A SR of PSA patient reported outcomes (PROs) was performed January 12017 and updated by hand search (22/11/2017). All articles assessing the measurement properties of the PSAID12 were reviewed. Strength of evidence was rated using COSMIN-OMERACT Good Methods checklist, and performance of measurement properties using the OMERACT standards ${ }^{1}$. We extracted data on domain match (face and content validity), construct validity, test-retest reliability, longitudinal construct validity, clinical trials discrimination, and thresholds of meaning.

Results: We identified six studies (129-474 patients in each study) assessing the measurement properties of the PSAID12 in adults with PSA. Domain match: PsAID12 was developed with 12 patient research partners, 139 patients who ranked domain importance and cognitive interviews with 65 patients. Construct validity: Three studies assessed correlation of the PSAID12 with PROs and clinical outcomes, and one study with two PROs. There was strong correlation with measures of function ( $n=3$ studies, $r>0.66)$, participation $(1, r>0.69)$, disease activity (2, r=0.64-0.87), and measures of pain, fatigue and stiffness (1 each, $r>0.83)$; moderate-strong with patient and physician global $(3, r=0.49-0.84)$; and moderate with $66 / 68$ joint counts $(1, r=0.4-0.57)$ and dactylitis $(1, r=0.49)$. Testretest reliability was high $(0.91(95 \% \mathrm{Cl} 0.87-0.94)$ and $0.95(0.92-0.96))$. Longitudinal construct validity was good with moderate to large standardized response mean (SRM) 0.74 ( $n=53$, changed therapy) and 0.91 ( $n=71$, changed therapy and rated themselves improved). The patient acceptable symptom state (PASS) was 4 in a single study. The minimal clinically important improvement (MCII) varied between 3 (original PsAID development study) and 1.25 (subsequent UK study). Conclusions: This review suggests there is evidence for excellent content validity and reliability and good construct validity and responsiveness of the PsAID12 questionnaire as a measure of HRQol in PsA. MCII and discrimination in clinical trials need to be defined.

\section{REFERENCE:}

1. Boers M, Kirwan JR, Tugwell $P$, et al. The OMERACT Handbook. Accessed 5 January 2018, https://www.omeract.org/resources

Disclosure of Interest: None declared DOI: 10.1136/annrheumdis-2018-eular.3040

\section{FRI0675 RABIOPRED, AN INNOVATIVE THERANOSTIC TOOL TO ASSIST CLINICIANS SELECT AN OPTIMAL ANTI-TNF ALPHA BIOLOGICAL THERAPY FOR RHEUMATOID ARTHRITIS PATIENTS}

S. Danilin ${ }^{1}$, E. Schordan ${ }^{1}$, M. Coq ${ }^{1}$, S. AIT ABBI NAZI ${ }^{1}$, M. Mehdi ${ }^{1}$, H. Firat ${ }^{1}, N$ Arber $^{2}$, V. Breuil ${ }^{3}$, A.-L. Demoux ${ }^{4}$, A. Gül ${ }^{5}$, J.-E. Gottenberg ${ }^{6}$, G. Hatemi ${ }^{5}$, T. Huizinga ${ }^{7}, \mathrm{~N}$. Inanç ${ }^{8}$, J. Vencovsky ${ }^{9}$, C. Jorgensen ${ }^{10}$ on behalf of RABIOPRED Consortium - Horizon 2020 SME Instrument program. ${ }^{1}$ FIRALIS, Huningue, France, ${ }^{2}$ Tel Aviv Sourasky Medical Center, Tel aviv, Israel, ${ }^{3} \mathrm{CHU}$ Nice, Nice, ${ }^{4} \mathrm{AP}$ HM, Marseille, France, ${ }^{5}$ Istanbul Medical University, Istanbul, Turkey, ${ }^{6} \mathrm{CHU}$ Strasbourg, Strasbourg, France, ${ }^{7}$ Leiden University Medical Center, Leiden, Netherlands, ${ }^{8}$ Marmara University, Istanbul, Turkey, ${ }^{9}$ Institute of Rheumatology, Prague, Czech Republic, ${ }^{10} \mathrm{CHU}$ Montpellier, Montpellier, France

Background: TNF alpha blockers form 2nd line treatment choice for Rheumatoid Arthritis (RA) patients. Up to $30 \%$ of RA patients do not respond to TNF alpha blockers for unknown reasons, causing a significant impact on patients' outcome and healthcare industry. Therefore, there is an unmet need for a tool to predict treatment response that could help clinicians to choose an optimal treatment for RA patients.

Objectives: By using Immuno-Detect, an innovative targeted gene sequencing panel of 2155 mRNA targets associated with immune-inflammatory pathways, we aimed to develop an algorithm, RABIOPRED, that predicts non-response to TNF alpha blockers.

Methods: Paxgene samples obtained at baseline from 68 patients naïve to TNF alpha blockers were directly profiled without extraction with Immuno-Detect panel on HTG EdgeSeq platform, a combination of a nuclease protection assay \& next generation sequencing (NGS). Patients were treated with Infliximab, Etanercept or Adalimumab and disease activity score was measured based on DAS28 score at 3 months. Response to treatment was assessed by categorizing the patients according to EULAR response criteria. Gene combinations were selected using variable importance score (VIS). Predictive modeling performance was evaluated using the area under the curve (AUC) and confusion matrix.

Results: Analytical validation of Immuno-Detect panel shows a very high reproducibility on Paxgene and extracted RNA samples with correlation factor of 0.975 and 0.96 respectively. In paxgene samples, among 2155 genes, 1172 mRNAs are significantly expressed with a mean CV of $9.77 \%$ (976 mRNAs and mean CV of $11.98 \%$ for RNA). Most expressed target represent only $5 \%$ of the total reads and only 20 targets are reaching $1 \%$ of total reads showing a very well balanced panel. Performance of our predictive model shows an AUC of 0.905 with 0.88 accuracy. Our algorithm predicts non-responders to TNF alpha blockers with the sensitivity of 0.78 and positive predictive value of 0.91 . This algorithm will be further validated within the ongoing RABIOPRED Proof-of-Performance study (ClinicalTrials.gov Identifier: NCT03016260) based on 720 patients treated by anti-TNF alpha drugs ( 5 originators \& 3 biosimilars) launched in December 2016.

Conclusions: We are showing that Immuno-Detect panel accurately measures mRNA expression using HTG-EdgeSeq NGS platform. This panel can be furthe used to build signatures to predict TNF alpha blocker's non-response. The algorithm obtained in the current study will be later on validated in a multi-centric proofof-performance clinical study.

Disclosure of Interest: None declared

DOI: 10.1136/annrheumdis-2018-eular.6236 